TITLE:

\title{
Vibrational and rotational structure and excited-state dynamics of pyrene.
}

\section{AUTHOR(S):}

Baba, Masaaki; Saitoh, Motohisa; Kowaka, Yasuyuki; Taguma, Kunio; Yoshida, Kazuto; Semba, Yosuke; Kasahara, Shunji; ... Ohshima, Yasuhiro; Hsu, Yen-Chu; Lin, Sheng Hsien

\section{CITATION:}

Baba, Masaaki ... [et al]. Vibrational and rotational structure and excited-state dynamics of pyrene.. The Journal of chemical physics 2009, 131(22): 224318.

\section{ISSUE DATE:}

2009-12-14

URL:

http://hdl.handle.net/2433/91263

RIGHT:

c 2009 American Institute of Physics. 


\title{
Vibrational and rotational structure and excited-state dynamics of pyrene
}

\author{
Masaaki Baba, ${ }^{1, a)}$ Motohisa Saitoh, ${ }^{1}$ Yasuyuki Kowaka, ${ }^{1}$ Kunio Taguma, ${ }^{1}$ Kazuto Yoshida, ${ }^{2}$ \\ Yosuke Semba, ${ }^{2}$ Shunji Kasahara, ${ }^{2}$ Takaya Yamanaka, ${ }^{3}$ Yasuhiro Ohshima, ${ }^{3}$ \\ Yen-Chu Hsu, ${ }^{4}$ and Sheng Hsien Lin ${ }^{4,5}$ \\ ${ }^{1}$ Division of Chemistry, Graduate School of Science, Kyoto University, Kyoto 606-8502, Japan \\ ${ }^{2}$ Molecular Photoscience Research Center, Kobe University, Nada-ku, Kobe 657-8501, Japan \\ ${ }^{3}$ Institute for Molecular Science, Okazaki 444-8585, Japan \\ ${ }^{4}$ Institute of Atomic and Molecular Science, Academic Sinica, Taipei, Taiwan 10617, Republic of China \\ ${ }^{5}$ Department of Applied Chemistry, National Chiau Tung University, Hsinchu, Taiwan 300, \\ Republic of China
}

(Received 15 June 2009; accepted 10 November 2009; published online 11 December 2009)

\begin{abstract}
Vibrational level structure in the $S_{0}{ }^{1} A_{g}$ and $S_{1}{ }^{1} B_{3 u}$ states of pyrene was investigated through analysis of fluorescence excitation spectra and dispersed fluorescence spectra for single vibronic level excitation in a supersonic jet and through referring to the results of $a b$ initio theoretical calculation. The vibrational energies are very similar in the both states. We found broad spectral feature in the dispersed fluorescence spectrum for single vibronic level excitation with an excess energy of $730 \mathrm{~cm}^{-1}$. This indicates that intramolecular vibrational redistribution efficiently occurs at small amounts of excess energy in the $S_{1}{ }^{1} B_{3 u}$ state of pyrene. We have also observed a rotationally resolved ultrahigh-resolution spectrum of the $0_{0}^{0}$ band. Rotational constants have been determined and it has been shown that the pyrene molecule is planar in both the $S_{0}$ and $S_{1}$ states, and that its geometrical structure does not change significantly upon electronic excitation. Broadening of rotational lines with the magnetic field by the Zeeman splitting of $M_{J}$ levels was very small, indicating that intersystem crossing to the triplet state is minimal. The long fluorescence lifetime indicates that internal conversion to the $S_{0}$ state is also slow. We conclude that the similarity of pyrene's molecular structure and potential energy curve in its $S_{0}$ and $S_{1}$ states is the main cause of the slow radiationless transitions. (C) 2009 American Institute of Physics. [doi:10.1063/1.3270136]
\end{abstract}

\section{INTRODUCTION}

Pyrene is a prototypical molecule of the polycyclic aromatic hydrocarbons (PAHs) group, and it has been the subject of extensive spectroscopic studies in its condensed phase. ${ }^{1-7}$ Ever since emission of pyrene was observed from the comet $\mathrm{P} / \mathrm{Halley}{ }^{8}$ the gaseous molecule has attracted the interest of many researchers, and several studies of the jetcooled pyrene molecule have been reported. ${ }^{9-13}$ Its vibrational and rotational structures, however, have not been satisfactorily analyzed yet, and more careful measurements of jet spectroscopy and ultrahigh-resolution spectroscopy are desired to accurately determine the energy level structure of the isolated pyrene molecule. Pyrene possesses $16 \pi$ electrons and is not subject to Hückel's $4 n+2$ rule, which states that cyclic planar molecules in which each atom has a $p$ orbital are aromatic if they contain $4 n+2 \pi$ electrons. It is of great interest to investigate pyrene's molecular structure in both the $S_{0}$ and $S_{1}$ states. In addition, it is worth noting that the nonradiative decay rate in the $S_{1}$ state is fairly slow. Fluorescence lifetime was reported to be very long $(1.4 \mu \mathrm{s}),{ }^{9}$ and fluorescence quantum yield is expected to be high although there has been no accurate measurement for the isolated molecule. The aim of the present study is to

\footnotetext{
${ }^{a)}$ Author to whom correspondence should be addressed. Electronic mail: baba@kuchem.kyoto-u.ac.jp.
}

estimate the molecular structure of pyrene and to elucidate the cause of its slow nonradiative decay processes.

The $S_{1}$ state is expressed as one of the nearly degenerate configurations of highest occupied molecular orbital-1 (HOMO-1) to lowest unoccupied molecular orbital (LUMO) and HOMO to LUMO+ 1 electronic excitations. The symmetry of these two states is identical $\left(B_{3 u}\right)$ and they strongly couple with configuration interaction. The $S_{1}{ }^{1} B_{3 u}$ state is one of the mixed states with symmetric linear combination and the energy becomes smaller in it than in that of the state of HOMO to LUMO electronic excitation $\left(S_{2}{ }^{1} B_{2 u}\right.$ state). This situation is similar to that of naphthalene and the $S_{1}$ state is designated by ${ }^{1} L_{b} .{ }^{14}$ Relatively strong $b_{1 g}$ vibronic bands could be seen in the fluorescence excitation spectrum, ${ }^{9-13}$ providing evidence of strong vibronic coupling between the $S_{1}{ }^{1} B_{3 u}$ and $S_{2}{ }^{1} B_{2 u}$ states. The $S_{1} \leftrightarrow S_{0}$ transition moment is small so that the radiative lifetime of the $S_{1}$ state is very long. The long fluorescence lifetime indicates that radiationless processes in the $S_{1}$ state of the isolated pyrene molecule such as predissociation, internal conversion (IC) to the ground state, and intersystem crossing (ISC) to the triplet state are all very slow.

We observed the fluorescence excitation spectrum of the $S_{1}{ }^{1} B_{3 u} \leftarrow S_{0}{ }^{1} A_{g}$ transition and dispersed fluorescence spectra for single vibronic level excitation of jet-cooled pyrene. The vibrational structures in the $S_{0}$ and $S_{1}$ states were analyzed referring to the results of ab initio calculation. Further, 
we observed the rotationally resolved ultrahigh-resolution spectrum of the $0_{0}^{0}$ band, and rotational constants have been accurately determined for both the $S_{0}$ and $S_{1}$ states. In this article, we present these experimental results and discuss the vibrational and rotational level structure, and excited-state dynamics of the isolated pyrene molecule.

\section{EXPERIMENTAL}

Pyrene- $h_{10}$ (Wako Chemical) was purified by vacuum sublimations and pyrene- $d_{10}$ (Wako Chemical) was used without further purification. The solid sample was heated to $180{ }^{\circ} \mathrm{C}$ in a stainless steel container. The vapor was mixed with Ar gas and the mixed gas was expanded in a highvacuum chamber through a pulsed nozzle (an automobile fuel injector) to generate a supersonic jet.

As a light source, we used a pulsed dye laser (Lambda Physik LPD $3000, \Delta E=0.1 \mathrm{~cm}^{-1}$ ) pumped by an excimer laser (Lambda Physik LPX 105i, 308 nm, 100 mJ). Fluorescence from excited molecules in a supersonic jet was collected with a lens to a photomultiplier (Hamamatsu R927) through a glass filter (Toshiba L42) to block scattered laser light. The output was processed with a storage oscilloscope (Iwatsu-Lecroy 9362) for single photon counting. The total photon number for ten laser shots was recorded as the fluorescence intensity using the LABVIEW system. The fluorescence excitation spectrum was observed by changing the wavelength of laser light. The dispersed fluorescence spectrum was observed using a scanning monochromator (Nikon $\mathrm{P} 250$ ). The resolution was $30 \mathrm{~cm}^{-1}$ and the accuracy of transition wavenumber was $\pm 6 \mathrm{~cm}^{-1}$.

The ultrahigh-resolution spectrum was observed using a cw single-mode ring Ti:sapphire laser (Coherent CR899-29, $\Delta E=0.0001 \mathrm{~cm}^{-1}$ ) pumped by a $\mathrm{Nd}^{3+}: \mathrm{YVO}_{4}$ laser (SpectraPhysics Millennia X, $532 \mathrm{~nm}, 10 \mathrm{~W}$ ). The output was fed into an enhancement cavity (Spectra-Physics WavetrainSC, LBO) for second harmonics generation. We obtained singlemode UV laser light with a power of $20 \mathrm{~mW}$. The UV laser light beam was directed across the supersonic jet at right angles, which was collimated by a skimmer $(2 \mathrm{~mm}$ orifice diameter) and a slit (1 mm width). The distance between the nozzle and the slit was $30 \mathrm{~cm}$. The residual Doppler width was estimated to be $0.0002 \mathrm{~cm}^{-1}$ under this condition. Fluorescence was focused to a photomultiplier (Hamamatsu R565) using a pair of a spherical mirror and an ellipsoidal mirror with $90 \%$ efficiency of the solid angle. The output was processed with a gated photon counter (Stanford Research SR400). We observed a sub-Doppler fluorescence excitation spectrum by recording the change of fluorescence intensity with the laser wavelength. We simultaneously recorded the transmitted light intensity of a stabilized etalon (Burleigh CFT500) as frequency marks and a Dopplerlimited absorption spectrum of the iodine in order to calibrate transition wavenumber of each rotational line. The transition wavenumber of an iodine spectral line was calibrated using the iodine atlas. ${ }^{15}$ The accuracies of relative and absolute wavenumbers were 0.0001 and $0.003 \mathrm{~cm}^{-1}$, respectively.

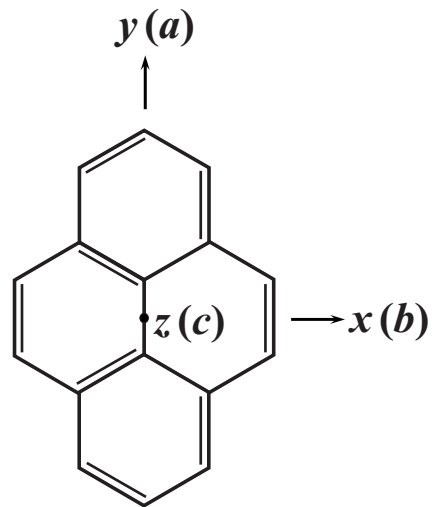

FIG. 1. Molecular structure and coordinate axes of pyrene.

\section{RESULTS AND DISCUSSION}

\section{A. Vibrational structure in the $S_{1}{ }^{1} B_{3 u}$ and $S_{0}{ }^{1} A_{g}$ states}

Pyrene is considered to be a planar molecule with $D_{2 h}$ symmetry in the $S_{0}{ }^{1} A_{g}$ and $S_{1}{ }^{1} B_{3 u}$ states, as we will show later in Sec. III B. In $D_{2 h}$ symmetry, the three coordinate axes are all equivalent because they possess one $C_{2}$, one $\sigma_{h}$, and two $\sigma_{v}$. Here, we define the axis system as shown in Fig. 1. Pyrene has 72 normal modes and its vibrational reducible representation is reduced as

$\Gamma=13 a_{g}+12 b_{1 g}+4 b_{2 g}+7 b_{3 g}+5 a_{u}+7 b_{1 u}+12 b_{2 u}+12 b_{3 u}$.

The vibrational energies in the excited state can be determined by excess energies of observed bands in a fluorescence excitation spectrum. Figure 2 shows the fluorescence excitation spectra of the $S_{1}{ }^{1} B_{3 u} \leftarrow S_{0}{ }^{1} A_{g}$ transition of pyrene- $h_{10}$ and pyrene- $d_{10}$ in supersonic jets. We could clearly resolve and identify even weak vibronic bands al-
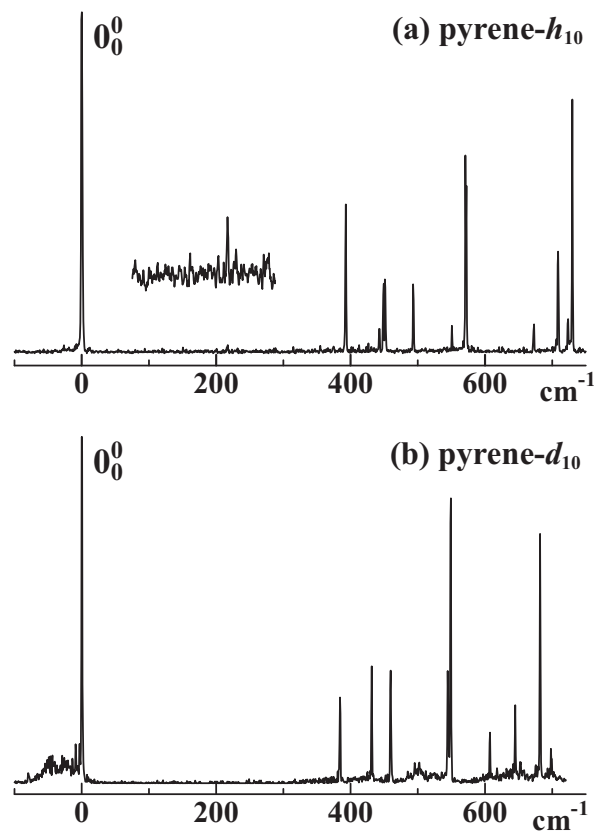

FIG. 2. Fluorescence excitation spectra of the $S_{1}{ }^{1} B_{3 u} \leftrightarrow S_{0}{ }^{1} A_{g}$ transition of (a) pyrene- $h_{10}$ and (b) pyrene- $d_{10}$ in supersonic jets. 
TABLE I. Vibrational energies $\left(\mathrm{cm}^{-1}\right)$ and assignments of the bands observed in the fluorescence excitation spectrum of the $S_{1}{ }^{1} B_{3 u} \leftarrow S_{0}{ }^{1} A_{g}$ transition of pyrene- $h_{10}$.

\begin{tabular}{|c|c|c|c|c|}
\hline $\begin{array}{l}\text { Band } \\
\text { number }\end{array}$ & $\begin{array}{l}\text { Vibrational } \\
\text { energy }\end{array}$ & Intensity $^{\mathrm{a}}$ & Band type & Assignment \\
\hline 1 & 0 & $\mathrm{~m}$ & $b$ & $0_{0}^{0}\left(27204 \mathrm{~cm}^{-1}\right)$ \\
\hline 2 & 217 & vw & & \\
\hline 3 & 232 & $\mathrm{vw}$ & & \\
\hline 4 & 324 & vw & & \\
\hline 5 & 374 & vw & & \\
\hline 6 & 393 & $\mathrm{~s}$ & $b$ & $13_{0}^{1}\left(a_{g}\right)$ \\
\hline 7 & 443 & w & $a$ & \\
\hline 8 & 449 & $\mathrm{~m}$ & $a$ & $25_{0}^{1}\left(b_{1 g}\right)$ \\
\hline 9 & 452 & $\mathrm{~m}$ & $a$ & \\
\hline 10 & 494 & $\mathrm{~m}$ & $a$ & $24_{0}^{1}\left(b_{1 g}\right)$ \\
\hline 11 & 551 & w & $a$ & \\
\hline 12 & 572 & $\mathrm{~s}$ & $b$ & $12_{0}^{1}\left(a_{g}\right)$ \\
\hline 13 & 673 & $\mathrm{~m}$ & $a$ & \\
\hline 14 & 709 & $\mathrm{~s}$ & $a$ & \\
\hline 15 & 724 & $\mathrm{~m}$ & $a$ & \\
\hline 16 & 730 & $\mathrm{~s}$ & $a$ & $23_{0}^{1}\left(b_{1 g}\right)$ \\
\hline 17 & 779 & $\mathrm{~m}$ & $b$ & \\
\hline 18 & 780 & vs & $b$ & \\
\hline 19 & 782 & vs & $b$ & \\
\hline 20 & 783 & $\mathrm{~s}$ & $b$ & \\
\hline 21 & 944 & w & & \\
\hline 22 & 967 & w & & \\
\hline 23 & 1023 & $\mathrm{w}$ & & $10_{0}^{1}\left(a_{g}\right)$ \\
\hline 24 & 1066 & w & & \\
\hline 25 & 1070 & w & & \\
\hline 26 & 1111 & $\mathrm{~m}$ & & $22_{0}^{1}\left(b_{1 g}\right)$ \\
\hline 27 & 1144 & $\mathrm{w}$ & & $9{ }_{0}^{1}\left(a_{g}\right)$ \\
\hline 28 & 1155 & w & & \\
\hline 29 & 1162 & w & & \\
\hline 30 & 1246 & w & & $20_{0}^{1}\left(b_{1 g}\right)$ \\
\hline 31 & 1250 & w & $b$ & $8_{0}^{1}\left(a_{g}\right)$ \\
\hline 32 & 1282 & w & & \\
\hline 33 & 1298 & w & & \\
\hline 34 & 1303 & w & & $23_{0}^{1} 12_{0}^{1}$ \\
\hline 35 & 1331 & w & $b$ & $7_{0}^{1}\left(a_{g}\right)$ \\
\hline 36 & 1354 & w & & $12_{0}^{1} 11_{0}^{1}$ \\
\hline 37 & 1357 & w & & $19_{0}^{1}\left(b_{1 g}\right)$ \\
\hline 38 & 1397 & $\mathrm{~m}$ & $b$ & $6_{0}^{1}\left(a_{g}\right)$ \\
\hline 39 & 1467 & $\mathrm{~m}$ & & $17_{0}^{1}\left(b_{1 g}\right)$ \\
\hline 40 & 1520 & w & & \\
\hline 41 & 1527 & $\mathrm{w}$ & & \\
\hline 42 & 1537 & w & & \\
\hline 43 & 1568 & w & & $11_{0}^{2}$ \\
\hline 44 & 1575 & $\mathrm{~m}$ & & $16_{0}^{1}\left(b_{1 g}\right)$ \\
\hline
\end{tabular}

"vw stands for "very weak," w for "weak," m for "medium," s for "strong," and vs for "very strong."

though the spectral features were almost identical to those reported before..$^{9-13}$ The excess energies of observed vibronic bands of pyrene- $h_{10}$ and pyrene- $d_{10}$ are listed in Tables I and II, respectively. In order to make reliable vibrational assignments, we observed the rotational envelopes of individual vibronic bands. The results are shown in Fig. 3. Pyrene is an asymmetric-top molecule and its rotational $a, b$, and $c$ axes are parallel to $y$ (long axis), $x$ (short axis), and $z$ (out of plane), respectively. In the $0_{0}^{0}$ band, two intensity maxima of $P$ and $R$ transitions are observed. This is the typical feature
TABLE II. Vibrational energies $\left(\mathrm{cm}^{-1}\right)$ and assignments of the bands observed in the fluorescence excitation spectrum of the $S_{1}{ }^{1} B_{3 u} \leftarrow S_{0}{ }^{1} A_{g}$ transition of pyrene- $d_{10}$.

\begin{tabular}{ccccc}
\hline \hline $\begin{array}{c}\text { Band } \\
\text { number }\end{array}$ & $\begin{array}{c}\text { Vibrational } \\
\text { energy }\end{array}$ & Intensity $^{\mathrm{a}}$ & Band type & Assignment \\
\hline 1 & 0 & $\mathrm{vs}$ & $b$ & $0_{0}^{0}\left(27283 \mathrm{~cm}^{-1}\right)$ \\
2 & 384 & $\mathrm{~m}$ & $b$ & $13_{0}^{1}\left(a_{g}\right)$ \\
3 & 431 & $\mathrm{~m}$ & $a$ & $25_{0}^{1}\left(b_{1 g}\right)$ \\
4 & 460 & $\mathrm{~m}$ & $a$ & $24_{0}^{1}\left(b_{1 g}\right)$ \\
5 & 495 & $\mathrm{vw}$ & & \\
6 & 502 & $\mathrm{vw}$ & & \\
7 & 544 & $\mathrm{~m}$ & $b$ & $12{ }_{0}^{1}\left(a_{g}\right)$ \\
8 & 549 & $\mathrm{~s}$ & $b$ & \\
9 & 607 & $\mathrm{w}$ & $a$ & $23{ }_{0}^{1}\left(b_{1 g}\right)$ \\
10 & 645 & $\mathrm{w}$ & $a$ & \\
11 & 682 & $\mathrm{~s}$ & $a$ & \\
12 & 698 & $\mathrm{w}$ & & \\
\hline \hline
\end{tabular}

${ }_{\mathrm{a} w}^{\mathrm{a}}$ stands for very weak, $\mathrm{w}$ for weak, $\mathrm{m}$ for medium, $\mathrm{s}$ for strong, and $\mathrm{vs}$ for very strong.

of a $b$-type transition in which the electronic transition moment is parallel to the $x$ axis. Consequently, the $S_{1}$ state can be identified as ${ }^{1} B_{3 u}$. We found several $a$-type vibronic bands in which a sharp $Q$ peak could be seen at the band center. The $a$-type band is identified as a transition to a $b_{1 g}$ vibrational level of which the transition moment is parallel to the $a(y)$ axis. The intensity arises from vibronic interaction with the $S_{2}{ }^{1} B_{2 u}$ state. ${ }^{16-19}$ The $b$-type vibronic band, in contrast, is identified as a transition to an $a_{g}$ vibrational level, the symmetry of which is the same as that of the $0_{0}^{0}$ band. Although the rotational envelope of a vibronic band observed at $780 \mathrm{~cm}^{-1}$ is strange, this band shape could be reproduced as an envelope of four partially overlapped $b$-type transitions. The vibrational energies in the $S_{0}$ state can be determined by the dispersed fluorescence spectrum for single vibronic level excitation. Figure 4 shows the dispersed fluorescence spectrum that occurs when the laser wavelength is tuned to the $S_{1} \leftarrow S_{0} 0_{0}^{0}$ band. The vibrational energies of observed bands are listed in Table III.

Accurate theoretical calculation is necessary for normal- (a) pyrene- $h_{10}$

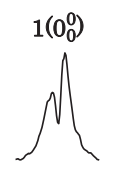

$\mu$
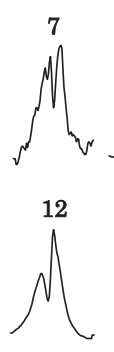

$\int_{0.1-1}^{14}$

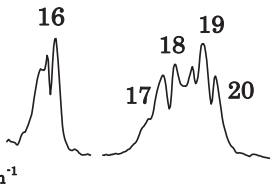

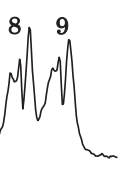

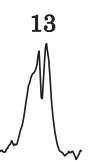

(b) pyrene- $d_{10}$
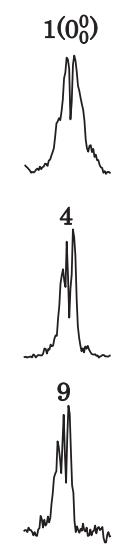
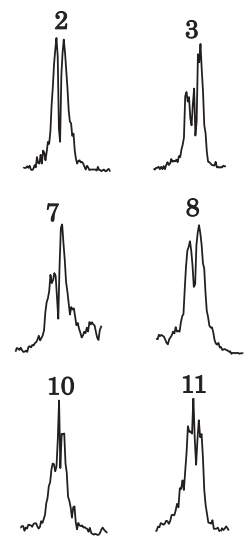

FIG. 3. Rotational envelopes of prominent bands in the fluorescence excitation spectra of the $S_{1}{ }^{1} B_{3 u} \leftarrow S_{0}{ }^{1} A_{g}$ transition of (a) pyrene- $h_{10}$ and (b) pyrene- $d_{10}$. 


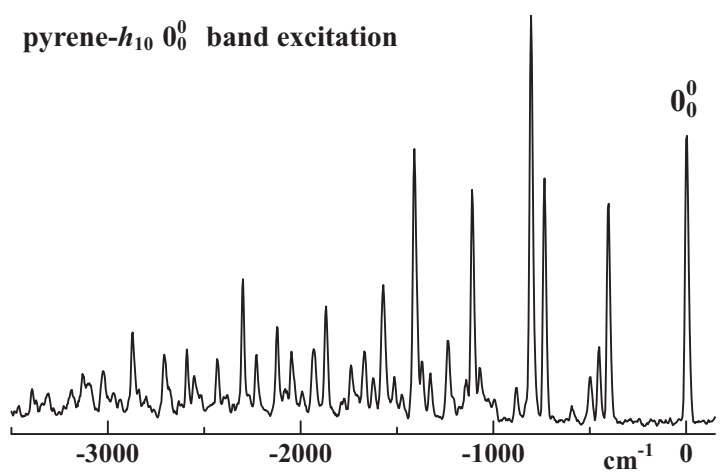

FIG. 4. Dispersed fluorescence spectrum of jet-cooled pyrene- $h_{10}$ for excitation of the $S_{1}{ }^{1} B_{3 u} \leftarrow S_{0}{ }^{1} A_{g} 0_{0}^{0}$ band. The resolution is $30 \mathrm{~cm}^{-1}$.

mode assignments of these observed vibronic bands. For the $S_{0}$ state, we calculated the vibrational energies using the density-functional-theory (DFT) method at the B3LYP/6$311 \mathrm{G}(\mathrm{d}, \mathrm{p})$ level with the GAUSSIAN 03 program package. ${ }^{20}$

TABLE III. Vibrational energies $\left(\mathrm{cm}^{-1}\right)$ and assignments of the bands observed in the dispersed fluorescence spectrum of the pyrene- $h_{10}$ for the $S_{1}{ }^{1} B_{3 u} \leftarrow S_{0}{ }^{1} A_{g} 0_{0}^{0}$ band excitation.

\begin{tabular}{|c|c|c|c|}
\hline $\begin{array}{l}\text { Band } \\
\text { number }\end{array}$ & $\begin{array}{c}\text { Vibrational } \\
\text { energy }\end{array}$ & Intensity $^{\mathrm{a}}$ & Assignment \\
\hline 1 & 0 & s & $0_{0}^{0}\left(27204 \mathrm{~cm}^{-1}\right)$ \\
\hline 2 & 402 & $\mathrm{~s}$ & $13_{1}^{0}\left(a_{g}\right)$ \\
\hline 3 & 451 & w & $25_{1}^{0}\left(b_{1 g}\right)$ \\
\hline 4 & 500 & $\mathrm{w}$ & $24_{1}^{0}\left(b_{1 g}\right)$ \\
\hline 5 & 593 & $\mathrm{vw}$ & $12_{1}^{0}\left(a_{g}\right)$ \\
\hline 6 & 735 & $\mathrm{~s}$ & $23_{1}^{0}\left(b_{1 g}\right)$ \\
\hline 7 & 805 & vs & $11_{1}^{0}\left(a_{g}\right)$ \\
\hline 8 & 883 & $\mathrm{w}$ & $25_{2}^{0}$ \\
\hline 9 & 999 & $\mathrm{vw}$ & $24_{2}^{0}$ \\
\hline 10 & 1072 & $\mathrm{w}$ & $10_{1}^{0}\left(a_{g}\right)$ \\
\hline 11 & 1111 & $\mathrm{~s}$ & $22_{1}^{0}\left(b_{1 g}\right)$ \\
\hline 12 & 1140 & vw & $9_{1}^{0}\left(a_{g}\right)$ \\
\hline 13 & 1212 & $\mathrm{w}$ & $21_{1}^{0}\left(b_{1 g}\right)$ \\
\hline 14 & 1238 & w & $8_{1}^{0}\left(a_{g}\right)$ and $20_{1}^{0}\left(b_{1 g}\right)$ \\
\hline 15 & 1326 & w & $7_{1}^{0}\left(a_{g}\right)$ \\
\hline 16 & 1372 & w & $19_{1}^{0}\left(b_{1 g}\right)$ \\
\hline 17 & 1410 & $\mathrm{~s}$ & $6_{1}^{0}\left(a_{g}\right)$ \\
\hline 18 & 1476 & vw & \\
\hline 19 & 1513 & $\mathrm{w}$ & $17_{1}^{0}\left(b_{1 g}\right)$ \\
\hline 20 & 1571 & $\mathrm{~m}$ & $16_{1}^{0}\left(b_{1 g}\right)$ \\
\hline 21 & 1624 & $\mathrm{w}$ & $4_{1}^{0}\left(a_{g}\right)$ \\
\hline 22 & 1670 & w & \\
\hline 23 & 1739 & w & \\
\hline 24 & 1867 & $\mathrm{~m}$ & \\
\hline 25 & 1936 & $\mathrm{w}$ & \\
\hline 26 & 2047 & $\mathrm{~m}$ & \\
\hline 27 & 2123 & $\mathrm{w}$ & \\
\hline 28 & 2228 & $\mathrm{w}$ & \\
\hline 29 & 2298 & $\mathrm{~m}$ & \\
\hline 30 & 2434 & $\mathrm{w}$ & \\
\hline 31 & 2552 & vw & \\
\hline 32 & 2590 & w & \\
\hline 33 & 2707 & w & \\
\hline 34 & 2875 & w & \\
\hline
\end{tabular}

$\overline{{ }^{\mathrm{a}} \mathrm{vw} \text { stands for very weak, } \mathrm{w} \text { for weak, } \mathrm{m} \text { for medium, } \mathrm{s} \text { for strong, and } \mathrm{vs}}$ for very strong.
For the $S_{1}{ }^{1} B_{3 u}$ state, we calculated the vibrational energies using the restricted configuration interaction of single excitation (RCIS) method with a $6-31 \mathrm{G}(\mathrm{d}, \mathrm{p})$ basis set. It is known that $a b$ initio calculation generally yields larger vibrational energies than those obtained experimentally, and an appropriate scaling factor was proposed for various calculational methods. For instance, 0.9757 was proposed for DFT(B3LYP)/6-311G**. ${ }^{21}$ In order to determine the best-fit scaling factor for pyrene in the $S_{0}$ state, we performed a least-squares fit and obtained 0.9830 . The scaled values are in good agreement with the observed vibrational energies. In the $S_{1}$ state, the vibrational energies are slightly different with symmetry because of interaction between the electronic states. We performed separate least-squares fits for the $a_{g}$ and $b_{1 g}$ bands, and obtained 0.8923 and 0.8848 , respectively. The scaled values are also in good agreement with the observed vibrational energies in the $S_{1}$ state. The resultant calculated and observed vibrational energies are listed in Table IV, and the vibronic assignments are shown in Tables I-III. The $780 \mathrm{~cm}^{-1}$ band is composed of four $b$-type bands. This band is considered to be Fermi multiplets due to the $11_{0}^{1}\left(a_{g}\right)$ fundamental band and the $13_{0}^{2}$ and $40_{0}^{2}$ overtone bands. Although these bands are supposed to be $b$-type, it is impossible to assign each observed band explicitly. The vibrational analysis in the $S_{0}$ and $S_{1}$ states were previously reported by Borisevich et al. ${ }^{11,12}$ based on the results of semiempirical $\mathrm{MO} / 8$ model calculation. We resolved the partially overlapped bands in the fluorescence excitation spectrum at the higher resolution. Accordingly, a number of vibrational energies are slightly different from theirs. Our assignments are based on the $a b$ initio theoretical calculation and the analysis of rotational envelope. The obtained vibrational energies are in good agreement with the observed vibronic energies. It should be noted that the vibrational energies in the $S_{1}$ state are very similar to those in the $S_{0}$ state. It indicates that the molecular structure and the potential energy curve are almost identical in these two states.

The $b_{1 g}$ bands are remarkably strong in the fluorescence excitation spectrum, suggesting that the vibronic interaction with the $S_{2}{ }^{1} B_{2 u}$ state is strong. The $S_{2} \leftarrow S_{0} 0_{0}^{0}$ band is observed at $31150 \mathrm{~cm}^{-1},{ }^{10}$ and the energy difference between the $S_{1}$ and $S_{2}$ states is only $3946 \mathrm{~cm}^{-1}$. Fluorescence lifetime becomes monotonously shorter as the excess energy becomes larger, and the lifetime at $4000 \mathrm{~cm}^{-1}$ is reported to be $\sim 400$ ns. $^{9}$ As the vibronic interaction between the $S_{1}$ and $S_{2}$ states becomes stronger, the radiative decay rate becomes larger because the $S_{2} \leftarrow S_{0}$ transition is significantly stronger. In a large molecule, intramolecular vibrational redistribution (IVR) is known to occur efficiently at high-vibrational levels of the $S_{1}$ state. We observed dispersed fluorescence spectra for excitation of prominent vibronic bands, and the results are shown in Fig. 5. Clearly, the broad emission component becomes stronger as the excess energy increases. These results indicate that the IVR process is remarkably fast at the vibrational levels over $730 \mathrm{~cm}^{-1}$ in the $S_{1}{ }^{1} B_{3 u}$ state of pyrene. It might be possible to estimate the IVR rate by the homogeneous width of a spectral line. However, it is ex- 
TABLE IV. Calculated and observed vibrational energies $\left(\mathrm{cm}^{-1}\right)$ of pyrene- $h_{10}$.

\begin{tabular}{|c|c|c|c|c|}
\hline Symmetry & Number & Vibration type & $\begin{array}{c}S_{0}{ }^{1} A_{g} \\
\text { Calc. }^{\mathrm{a}}(\text { Obs. })^{\mathrm{b}}\end{array}$ & $\begin{array}{c}S_{1}{ }^{1} B_{3 u} \\
\text { Calc. }^{\mathrm{c}}(\text { Obs. })^{\mathrm{d}}\end{array}$ \\
\hline \multirow{13}{*}{$a_{g}$} & 1 & $\mathrm{C}-\mathrm{H}$ stretch & 3143 & 3027 \\
\hline & 2 & $\mathrm{C}-\mathrm{H}$ stretch & 3134 & 3011 \\
\hline & 3 & $\mathrm{C}-\mathrm{H}$ stretch & 3118 & 2995 \\
\hline & 4 & $\mathrm{C}-\mathrm{C}$ stretch & $1646(1624)$ & 1661 \\
\hline & 5 & $\mathrm{C}-\mathrm{C}$ stretch $+\mathrm{C}-\mathrm{H}$ bend & 1572 & 1523 \\
\hline & 6 & $\mathrm{C}-\mathrm{C}$ stretch & $1406(1410)$ & $1420(1397)$ \\
\hline & 7 & Ring deform & $1333(1326)$ & $1313(1331)$ \\
\hline & 8 & Ring deform $+\mathrm{C}-\mathrm{H}$ bend & $1245(1238)$ & $1252(1250)$ \\
\hline & 9 & $\mathrm{C}-\mathrm{H}$ bend & $1152(1140)$ & 1139 (1144) \\
\hline & 10 & Ring deform $+\mathrm{C}-\mathrm{H}$ bend & $1076(1072)$ & $1033(1023)$ \\
\hline & 11 & Ring deform & $804(805)$ & $780(783)$ \\
\hline & 12 & Ring breathing & $587(593)$ & $556(572)$ \\
\hline & 13 & Ring deform & $406(402)$ & $395(393)$ \\
\hline \multirow[t]{12}{*}{$b_{1 g}$} & 14 & $\mathrm{C}-\mathrm{H}$ stretch & 3126 & 2978 \\
\hline & 15 & $\mathrm{C}-\mathrm{H}$ stretch & 3115 & 2964 \\
\hline & 16 & $\mathrm{C}-\mathrm{C}$ stretch & $1603(1571)$ & $1574(1575)$ \\
\hline & 17 & $\mathrm{C}-\mathrm{C}$ stretch $+\mathrm{C}-\mathrm{H}$ bend & $1513(1513)$ & $1474(1467)$ \\
\hline & 18 & $\mathrm{C}-\mathrm{C}$ stretch & 1415 & 1425 \\
\hline & 19 & $\mathrm{C}-\mathrm{H}$ bend+ring deform & $1381(1372)$ & 1397 (1357) \\
\hline & 20 & $\mathrm{C}-\mathrm{H}$ bend+ring deform & $1247(1238)$ & $1235(1246)$ \\
\hline & 21 & $\mathrm{C}-\mathrm{H}$ bend & $1183(1212)$ & 1158 \\
\hline & 22 & $\mathrm{C}-\mathrm{H}$ bend + ring deform & $1112(1111)$ & $1098(1111)$ \\
\hline & 23 & Ring deform & $739(735)$ & $724(730)$ \\
\hline & 24 & Ring deform & $501(500)$ & $490(494)$ \\
\hline & 25 & Ring deform & $456(451)$ & 446 (449) \\
\hline \multirow[t]{4}{*}{$b_{2 g}$} & 26 & oop $\mathrm{C}-\mathrm{H}$ bend & 905 & 917 \\
\hline & 27 & oop $\mathrm{C}-\mathrm{H}$ bend & 803 & 808 \\
\hline & 28 & oop ring deform & 530 & 503 \\
\hline & 29 & oop ring torsion & 243 & 225 \\
\hline \multirow[t]{7}{*}{$b_{3 g}$} & 30 & oop $\mathrm{C}-\mathrm{H}$ bend & 978 & 1011 \\
\hline & 31 & oop $\mathrm{C}-\mathrm{H}$ bend & 963 & 966 \\
\hline & 32 & oop $\mathrm{C}-\mathrm{H}$ bend $+\mathrm{C}-\mathrm{C}$ bend & 806 & 825 \\
\hline & 33 & oop C-H bend & 759 & 747 \\
\hline & 34 & oop ring deform & 571 & 507 \\
\hline & 35 & oop ring deform & 500 & 483 \\
\hline & 36 & oop ring wagging & 258 & 238 \\
\hline \multirow[t]{5}{*}{$a_{u}$} & 37 & oop $\mathrm{C}-\mathrm{H}$ bend & 968 & 1006 \\
\hline & 38 & oop $\mathrm{C}-\mathrm{H}$ bend & 891 & 900 \\
\hline & 39 & oop $\mathrm{C}-\mathrm{H}$ bend & 675 & 655 \\
\hline & 40 & oop ring deform & 395 & 378 \\
\hline & 41 & oop ring deform & 149 & 150 \\
\hline \multirow[t]{7}{*}{$b_{1 u}$} & 42 & oop $\mathrm{C}-\mathrm{H}$ bend & 969 & 976 \\
\hline & 43 & oop $\mathrm{C}-\mathrm{H}$ bend & 844 & 847 \\
\hline & 44 & oop $\mathrm{C}-\mathrm{H}$ bend & 746 & 742 \\
\hline & 45 & oop ring deform & 710 & 680 \\
\hline & 46 & oop ring deform & 493 & 449 \\
\hline & 47 & oop ring deform & 209 & 197 \\
\hline & 48 & Butterfly & 96 & 90 \\
\hline \multirow[t]{5}{*}{$b_{2 u}$} & 49 & C-H stretch & 3142 & 3026 \\
\hline & 50 & $\mathrm{C}-\mathrm{H}$ stretch & 3118 & 2997 \\
\hline & 51 & $\mathrm{C}-\mathrm{H}$ stretch & 3114 & 2990 \\
\hline & 52 & $\mathrm{C}-\mathrm{C}$ stretch & 1612 & 1571 \\
\hline & 53 & $\mathrm{C}-\mathrm{H}$ bend $+\mathrm{C}-\mathrm{C}$ stretch & 1463 & 1440 \\
\hline
\end{tabular}


TABLE IV. (Continued.)

\begin{tabular}{|c|c|c|c|c|}
\hline Symmetry & Number & Vibration type & $\begin{array}{c}S_{0}{ }^{1} A_{g} \\
\text { Calc. }^{\mathrm{a}}(\text { Obs. })^{\mathrm{b}}\end{array}$ & $\begin{array}{c}S_{1}{ }^{1} B_{3 u} \\
\text { Calc. }^{\mathrm{c}}(\text { Obs. })^{\mathrm{d}}\end{array}$ \\
\hline & 54 & $\mathrm{C}-\mathrm{H}$ bend + ring deform & 1439 & 1368 \\
\hline & 55 & $\mathrm{C}-\mathrm{H}$ bend + ring deform & 1251 & 1240 \\
\hline & 56 & Ring deform & 1099 & 1059 \\
\hline & 57 & Ring deform & 1001 & 959 \\
\hline & 58 & Ring deform & 823 & 789 \\
\hline & 59 & Ring deform & 694 & 671 \\
\hline & 60 & Ring deform & 500 & 483 \\
\hline \multirow[t]{12}{*}{$b_{3 u}$} & 61 & $\mathrm{C}-\mathrm{H}$ stretch & 3134 & 3011 \\
\hline & 62 & $\mathrm{C}-\mathrm{H}$ stretch & 3125 & 3003 \\
\hline & 63 & $\mathrm{C}-\mathrm{H}$ bend $+\mathrm{C}-\mathrm{C}$ stretch & 1621 & 1554 \\
\hline & 64 & $\mathrm{C}-\mathrm{H}$ bend $+\mathrm{C}-\mathrm{C}$ stretch & 1493 & 1519 \\
\hline & 65 & $\mathrm{C}-\mathrm{H}$ bend $+\mathrm{C}-\mathrm{C}$ stretch & 1438 & 1437 \\
\hline & 66 & $\mathrm{C}-\mathrm{H}$ bend $+\mathrm{C}-\mathrm{C}$ stretch & 1325 & 1305 \\
\hline & 67 & $\mathrm{C}-\mathrm{H}$ bend+ring deform & 1215 & 1202 \\
\hline & 68 & $\mathrm{C}-\mathrm{H}$ bend+ring deform & 1189 & 1162 \\
\hline & 69 & $\mathrm{C}-\mathrm{H}$ bend & 1150 & 1126 \\
\hline & 70 & $\mathrm{C}-\mathrm{H}$ bend+ring deform & 967 & 928 \\
\hline & 71 & Ring deform & 547 & 528 \\
\hline & 72 & Ring deform & 354 & 348 \\
\hline
\end{tabular}

${ }^{\mathrm{a}}$ Results of B3LYP/6-311G(d,p) are scaled by 0.9830 .

${ }^{\mathrm{b}}$ Determined using the dispersed fluorescence spectrum with an accuracy of $\pm 6 \mathrm{~cm}^{-1}$.

${ }^{\mathrm{c}}$ Results of RCIS/6-31G(d,p) are scaled by 0.8848 for the $b_{1 g}$ modes and 0.8923 for other modes.

${ }^{\mathrm{d}}$ Determined using the fluorescence excitation spectrum with an accuracy of $\pm 1 \mathrm{~cm}^{-1}$.

pected to be very small in the $S_{1}$ state of pyrene, and timeresolved fluorescence measurements are necessary to determine the absolute value.

\section{B. Rotational structure in the $S_{1}{ }^{1} B_{3 u}$ and $S_{0}{ }^{1} A_{g}$ states}

We observed a rotationally resolved ultrahigh-resolution fluorescence excitation spectrum of the $S_{1}{ }^{1} B_{3 u} \leftarrow S_{0}{ }^{1} A_{g} 0_{0}^{0}$ band of jet-cooled pyrene. The whole spectrum is shown in Fig. 6. The linewidth of an observed rotational line was $0.0003 \mathrm{~cm}^{-1}$, which was mainly due to the residual Doppler width of the collimated supersonic jet. The lifetime at the zero-vibrational level of the $S_{1}$ state was reported to be

(a) b-type band excitation

(b) a-type band excitation

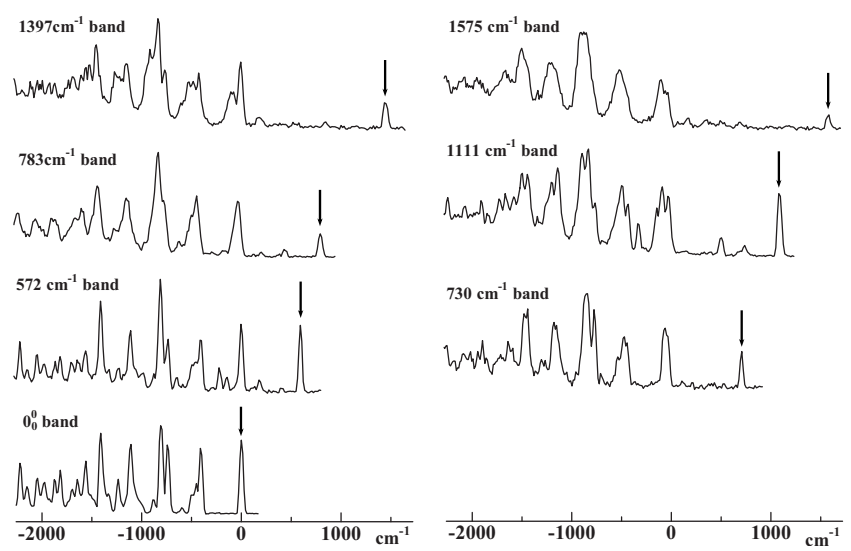

FIG. 5. Dispersed fluorescence spectra of jet-cooled pyrene- $h_{10}$ for excitation of the $S_{1}{ }^{1} B_{3 u} \leftarrow S_{0}{ }^{1} A_{g}$ prominent bands.
$1.4 \mu \mathrm{s}$, and natural linewidth is expected to be $0.000004 \mathrm{~cm}^{-1}$. The rotational temperature was estimated to be $12 \mathrm{~K}$ by the analysis of intensity distribution.

We also analyzed the rotational structure of pyrene using the $A$-reduced Hamiltonian method on an asymmetric-top molecule presented by Watson. ${ }^{22}$ The nonvanishing matrix elements are given by

$$
\begin{aligned}
\langle J K M| & H_{r}^{(A)}|J K M\rangle \\
= & \frac{1}{2}(B+C) J(J+1)+\left[A-\frac{1}{2}(B+C)\right] K^{2}-\Delta_{J} J^{2}(J+1)^{2} \\
& -\Delta_{J K} J(J+1) K^{2}-\Delta_{K} K^{4}
\end{aligned}
$$

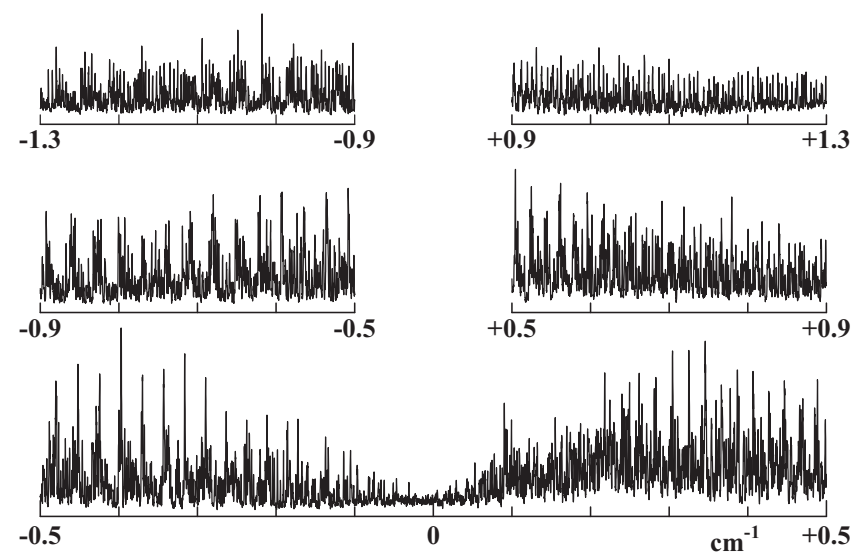

FIG. 6. Rotationally resolved fluorescence excitation spectrum of the $S_{1}{ }^{1} B_{3 u} \leftarrow S_{0}{ }^{1} A_{g} 0_{0}^{0}$ transition of pyrene- $h_{10}$. 


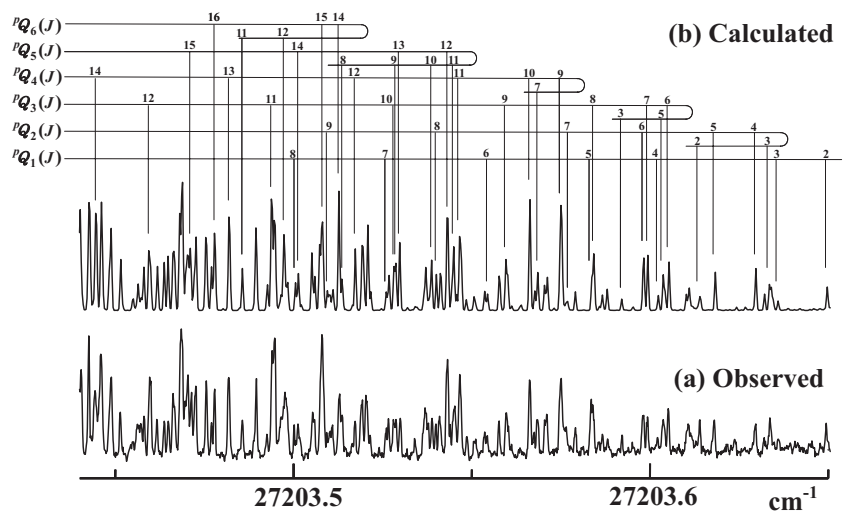

FIG. 7. (a) A part of the rotationally resolved fluorescence excitation spectrum of the $S_{1}{ }^{1} B_{3 u} \leftarrow S_{0}{ }^{1} A_{g} 0_{0}^{0}$ transition of pyrene- $h_{10}$ is compared to (b) the calculated spectrum using the obtained constants shown in Table V.

$$
\begin{aligned}
\langle J K & \left. \pm 2 M\left|H_{r}^{(A)}\right| J K M\right\rangle=\left\{\frac{1}{4}(B-C)-\delta_{J} J(J+1)\right. \\
& \left.-\frac{1}{2} \delta_{K}\left[(K \pm 2)^{2}+K^{2}\right]\right\}\{[J(J+1) \\
& -K(K \pm 1)][J(J+1)-(K \pm 1)(K \pm 2)]\}^{1 / 2},
\end{aligned}
$$

where $|J K M\rangle$ is the eigenfunction of a symmetric-top molecule. $J$ is the quantum number of total angular momentum $\boldsymbol{J}$, while $K$ and $M$ are quantum numbers of the projection of $\boldsymbol{J}$ along the molecule-fixed $a(y)$ axis and the space-fixed $Z$ axis, respectively. $A, B$, and $C$ are rigid-rotor rotational constants. $\Delta_{J}, \Delta_{J K}$, and $\Delta_{K}$ are symmetric-top quartic centrifugal distortion constants, and $\delta_{J}$ and $\delta_{K}$ are asymmetric-top distortion constants.

All rotational lines show $b$-type selection rules. The ${ }^{q} Q$ lines $\left(\Delta K_{a}=0, \Delta J=0\right)$ could not be seen around the band center, and the $S_{1}$ state has been confirmed to be ${ }^{1} B_{3 u}$. A part of the expanded spectrum in the $P$ branch region is shown in Fig. 7. The intensity ratio with nuclear spin multiplicity is calculated to be $e e: e o: o o: o e=A_{1}: B_{3}: B_{2}: B_{1}=18: 15: 16: 15$. We assigned 1510 rotational lines and obtained rotational constants of the $S_{0}$ and $S_{1}$ states. The results are tabulated in Table V. Although the spectrum is congested with many lines, we were able to assign most of them unambiguously. The transition energies calculated using the obtained rotational constants are in good agreement with the observed values and we could not find any energy shifts occurring through local perturbation. The calculated spectrum using the obtained rotational constants is compared to the observed one in Fig. 7.

The obtained rotational constants in the $S_{0}$ state $(A$ $=0.0339147, B=0.0186550$, and $C=0.0120406 \mathrm{~cm}^{-1}$ ) are similar to those obtained by ab initio calculation retaining $D_{2 h}$-symmetry $(A=0.0337674, B=0.0185557$, and $C$ $\left.=0.0119751 \mathrm{~cm}^{-1}\right)$. Inertial defect $\Delta=I_{c}-I_{b}-I_{a}$ is negligibly small in both the $S_{0}$ and $S_{1}$ states. Therefore, we conclude that the pyrene molecule is planar and $D_{2 h}$-symmetrical. Pyrene is not considered to be completely aromatic because it has $16 \pi$ electrons. However, the isolated pyrene molecule has been found to be highly symmetrical with three orthogonal twofold axes and symmetry planes. It is worth noting that the rotational constants in the $S_{1}{ }^{1} B_{3 u}$ state are very similar to those in the $S_{0}{ }^{1} A_{g}$ state. The differ-
TABLE V. Molecular constants of the $S_{0}{ }^{1} A_{g}\left(v^{\prime \prime}=0\right)$, and $S_{1}{ }^{1} B_{3 u}\left(v^{\prime}=0\right)$ states of pyrene- $h_{10}$. Rotational constants, band origin $T_{0}$, and standard deviation $\sigma$ are in units of $\mathrm{cm}^{-1}$. Moments of inertia $I_{a}, I_{b}$, and $I_{c}$ and inertial defect $\Delta$ are in units of $\mathrm{kg} \mathrm{m}^{2}$.

\begin{tabular}{llc}
\hline \hline Pyrene- $h_{10}$ & $S_{0}{ }^{1} A_{g}\left(v^{\prime \prime}=0\right)$ & $S_{1}{ }^{1} B_{3 u}\left(v^{\prime}=0\right)$ \\
\hline$A$ & $0.0339147(45)$ & $0.0336223(44)$ \\
$B$ & $0.0186550(32)$ & $0.0184864(32)$ \\
$C$ & $0.0120406(24)$ & $0.0119329(23)$ \\
$D_{K}\left(\times 10^{-8}\right)$ & $-2.8(12)$ & $-1.9(11)$ \\
$D_{J K}\left(\times 10^{-8}\right)$ & $3.88(67)$ & $2.99(68)$ \\
$D_{J}\left(\times 10^{-9}\right)$ & $-8.2(18)$ & $-7.0(18)$ \\
$\delta_{K}\left(\times 10^{-8}\right)$ & $-6.4(10)$ & $-5.25(96)$ \\
$\delta_{J}\left(\times 10^{-9}\right)$ & $-4.24(81)$ & $-3.53(79)$ \\
$\kappa$ & -0.39523 & -0.39570 \\
$I_{a}\left(\times 10^{-46}\right)$ & 82.539 & 83.256 \\
$I_{b}\left(\times 10^{-46}\right)$ & 150.05 & 151.42 \\
$I_{c}\left(\times 10^{-46}\right)$ & 232.49 & 234.58 \\
$\Delta\left(\times 10^{-46}\right)$ & -0.10730 & -0.09534 \\
$T_{0}$ & $\ldots$ & $27203.680(3)$ \\
$\sigma$ & $\ldots$ & 0.00034 \\
Fit lines & $\ldots$ & 1510 \\
Band type & & $b$ \\
\hline \hline
\end{tabular}

ences are less than $1 \%$ for all the $A, B$, and $C$ values. This indicates that structural change upon electronic excitation is very small.

\section{Excited-state dynamics in the $S_{1}{ }^{1} B_{3 u}$ state}

$\pi$ orbitals of pyrene calculated at the B3LYP/6-31G(d,p) level are shown in Fig. 8. $\phi_{53}(\mathrm{HOMO})$ and $\phi_{54}(\mathrm{LUMO})$ are uniformly distributed over each whole $\pi$ bonds although no population is present at the molecular center. The $S_{2}{ }^{1} B_{2 u}$ state is approximately expressed by the configuration of $\mathrm{HOMO} \rightarrow$ LUMO excitation. The wave functions of $\phi_{52}(\mathrm{HOMO}-1)$ and $\phi_{55}(\mathrm{LUMO}+1)$ are also uniform. The $S_{1}{ }^{1} B_{3 u}$ state is expressed by the almost equally mixed configuration of $\phi_{52}(\mathrm{HOMO}-1) \rightarrow \phi_{54}(\mathrm{LUMO})$ and

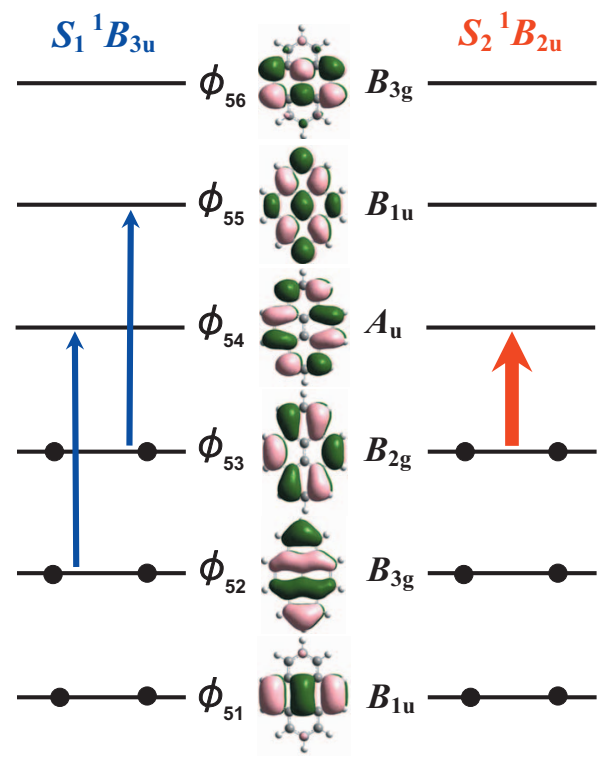

FIG. 8. $\pi$ molecular orbitals of pyrene calculated at the B3LYP/6-311G(d,p) level. 
$\phi_{53}(\mathrm{HOMO}) \rightarrow \phi_{55}(\mathrm{LUMO}+1)$ electronic excitations. The $\pi$ bond order is defined by the sum over all $\pi$ electrons of products of atomic orbital coefficients. Although the number of nodes in the wave function increases by two as a result of electronic excitation, the change in electronic distribution is small. The change in the bond order is further diluted among 16 electrons. Consequently, structural change resulting from electronic excitation is expected to be small.

We demonstrate that the excited-state dynamics in the $S_{1}$ state of pyrene is closely related to its geometrical structure. The fluorescence lifetime of the $S_{1}$ state of pyrene is very long, which indicates that all the radiationless transitions are very slow. Three processes that may be involved are predissociation, ISC to the triplet state, and IC to the $S_{0}$ state. The bond energies are significantly higher than the excitation energy of the $S_{1} \rightarrow S_{0}$ transition so that predissociation does not occur at the zero-vibrational level in the $S_{1}$ state of pyrene. ISC is not expected to be efficient, according to El-Sayed's rule that spin-orbit interaction between the ${ }^{1} \pi \pi^{*}$ and ${ }^{3} \pi \pi^{*}$ states is very weak for a planar molecule. ${ }^{23}$ In order to confirm the triplet character, we observed broadening of a rotational line with the magnetic field, which is caused by the Zeeman splitting of $M_{J}$ levels. The observed broadening was considerably small even at a magnetic field of $1.0 \mathrm{~T}$. Accordingly, we conclude that the triplet mixing is minimal and that ISC to the triplet state is very slow.

Next we discuss the inactivity of IC in the $S_{1}{ }^{1} B_{3 u}$ state of the isolated pyrene molecule. The IC to the $S_{0}$ state is caused by vibronic interaction. The energy difference between the zero-vibrational levels of the $S_{0}$ and $S_{1}$ states is $27204 \mathrm{~cm}^{-1}$, and the vibrational quantum number of the $S_{0}$ state is large in the $S_{1}$ energy region. The vibronic interaction is considered to be very weak when the equilibrium molecular structure and potential energy curves are approximately the same for both the $S_{0}$ and $S_{1}$ states.

The IC rate is given by ${ }^{24-27}$

$$
\begin{aligned}
W_{I C} & \propto \sum_{i}\left|\left\langle\phi_{S_{0}}\left|\frac{\partial}{\partial Q_{i}}\right| \phi_{S_{1}}\right\rangle\right|^{2}\left|\left\langle\chi_{S_{0}}^{v^{\prime \prime}}\left|\frac{\partial}{\partial Q_{i}}\right| \chi_{S_{1}}^{v^{\prime}=0}\right\rangle\right|^{2} \delta\left(E_{S_{1}}^{v^{\prime}=0}-E_{S_{0}}^{v^{\prime \prime}}\right) \\
& \approx \frac{\sum_{i}\left|\left\langle\phi_{S_{0}}\left(r, Q_{0}\right)\left|\left(\frac{\partial U(r, Q)}{\partial Q_{i}}\right)_{Q_{0}}\right| \phi_{S_{1}}\left(r, Q_{0}\right)\right\rangle\right|}{\left(E_{S_{1}}\left(Q_{0}\right)-E_{S_{0}}\left(Q_{0}\right)\right)^{2}}\left|\left\langle\chi_{S_{0}}^{v^{\prime \prime}}\left(Q_{i}\right)\left|\frac{\partial}{\partial Q_{i}}\right| \chi_{S_{1}}^{v^{\prime}=0}\left(Q_{i}\right)\right\rangle \prod_{j \neq i}\left\langle\chi_{S_{0}}^{v^{\prime \prime}}\left(Q_{j}\right) \mid \chi_{S_{1}}^{v^{\prime}=0}\left(Q_{j}\right)\right\rangle\right|^{2} \delta\left(E_{S_{1}}^{v^{\prime}=0}-E_{S_{0}}^{v^{\prime \prime}}\right) .
\end{aligned}
$$

Here, $\phi_{S_{0}}$ and $\phi_{S_{1}}$ represent the electronic wave functions of the $S_{0}$ and $S_{1}$ states, respectively. $\chi_{S_{0}}^{v^{\prime \prime}}$ is the vibrational wave function of the high-vibrational level in the $S_{0}$ state and $\chi_{S_{1}{ }^{\prime}=0}$ is that of the zero-vibrational level in the $S_{1}$ state. $\delta$ is the Dirac delta function and $Q_{i}$ is a normal coordinate. The second and third matrix elements are nonadiabatic FranckCondon overlap of a promoting mode and Franck-Condon overlap of other modes, respectively. In $D_{2 h}$ symmetry, the $S_{1}{ }^{1} B_{3 u}$ state couples with the $S_{0}$ state through a $b_{3 u}$ vibration. The energy gap between the $S_{0}$ and $S_{1}$ states is large and their molecular structures are similar. The amplitude of wave function of the $S_{1}$ zero-vibrational level is large at the equilibrium nuclear positions (potential bottom), whereas that of the $S_{0}$ high-vibrational level is large near both edges of the potential energy curve. According to energy gap law, ${ }^{24}$ the vibrational overlap is very small if the potential energy curves are approximately the same for the $S_{0}$ and $S_{1}$ states and the energy gap is large. We have shown that the equilibrium molecular structure is almost identical by the determined rotational constants and that the slope of the potential energy curve is also very similar by the observed vibrational energy. Therefore, the IC process in the isolated pyrene molecule is presumed to be very slow. Remarkably fast radiationless transitions such as ISC and IC with the quantum yields of 0.37 (Ref. 28) and $0.71,{ }^{29}$ respectively, were observed in condensed phases. These processes are attributed to structural change due to intermolecular interaction with the environment. The rates of radiationless transitions are considered to be strongly dependent on the molecular structure and potential energy curves so that it is noteworthy to compare the vibrational energies of an isolated molecule with those in condensed phases. For $a_{g}$ modes, the vibrational energies are approximately the same for gas and condensed phases, as shown in Table VI. ${ }^{1,5,30,31}$ Although the observed vibrational energies in the $S_{0}$ state are similar for gas and condensed phases, those in the $S_{1}$ state are appreciably different, supporting that the potential energy curves are affected by intermolecular interaction. Such slow nonradiative decay in the $S_{1}{ }^{1} B_{3 u}$ state is supposed to be common for large PAHs, in which the molecular structure is not significantly changed upon electronic excitation. Predissociation does not occur because the $S_{1}$ excitation energy is less than the bond energies. The ISC to the triplet state scarcely occurs according to El-Sayed's rule. The IC rate is considered to be strongly correlated with the geometrical structure. The structural change upon $S_{1} \leftarrow S_{0}$ excitation is small in large PAH molecules. In such cases, the overlap of vibrational wave functions is expected to be small between high-vibrational levels of the $S_{0}$ state and the zero-vibrational level of the $S_{1}$ state. Consequently, the vibronic coupling becomes very weak and the IC in the $S_{1}$ state is expected to be slow.

We assume that IC is relatively faster in the $B_{2 u}$ state of a PAH molecule with $D_{2 h}$ symmetry. For instance, the fluo- 
TABLE VI. Observed $a_{g}$ vibrational energies of pyrene- $h_{10}$ for gas and condensed phases.

\begin{tabular}{ccccc}
\hline \hline Mode number & Gas $^{\mathrm{a}}$ & In biphenyl $^{\mathrm{b}}$ & In heptane $^{\mathrm{c}}$ & Crystal $^{\mathrm{d}}$ \\
\hline 5 & \multicolumn{5}{c}{${ }^{1}{ }^{1} A_{g}$} & & \\
6 & 1571 & 1552 & 1550 & 1552 \\
7 & 1410 & 1408 & 1405 & 1408 \\
8 & 1326 & 1355 & & 1352 \\
9 & 1238 & 1240 & 1236 & 1243 \\
10 & 1140 & 1144 & & \\
11 & 1072 & 1063 & 1065 & 1067 \\
12 & 805 & 801 & 801 & 805 \\
13 & 593 & 596 & 588 & 593 \\
& 402 & 408 & 405 & 408 \\
5 & & & & \\
6 & 1527 & $S_{1}{ }^{1} B_{3 u}$ & & \\
7 & 1397 & 1452 & 1444 & 1450 \\
8 & 1303 & 1339 & 1328 & 1322 \\
9 & 1250 & 1249 & 1245 & 1237 \\
10 & 1144 & & & \\
11 & 1023 & 1030 & 1029 & 1030 \\
12 & 783 & 782 & 779 & \\
13 & 572 & 583 & 573 & 570 \\
\hline \hline
\end{tabular}

${ }^{\mathrm{a}}$ Present work.

${ }^{\mathrm{b}}$ Reference 1 .

${ }^{\mathrm{c}}$ Reference 5 .

${ }^{\mathrm{d}}$ Crystal from Ref. 30 for the $S_{0}{ }^{1} A_{g}$ state and in hexane from Ref. 31 for the $S_{1}{ }^{1} B_{3 u}$ state.

rescence lifetime of anthracene- $d_{10}(4 \mathrm{~ns})$ is much shorter than that of anthracene- $\left.h_{10}(18 \mathrm{~ns})\right)^{32,33}$ This fact suggests that its promoting mode is $\mathrm{C}-\mathrm{H}$ stretching, which includes nuclear motion along the $y$ axis, that is, $b_{2 u}$ vibration. The IC rate in the ${ }^{1} B_{3 u}$ state, which couples with the $S_{0}{ }^{1} A_{g}$ state through a $b_{3 u}$ vibrational mode, is presumed to be slow probably because of the small nonadiabatic Franck-Condon overlap. The observed fluorescence lifetimes of naphthalene $(\sim 300 \mathrm{~ns})$ and pyrene $(\sim 1400 \mathrm{~ns})$ are long in contrast to the short lifetimes of the $S_{1}{ }^{1} B_{2 u}$ states of anthracene (18 ns), tetracene $(20 \mathrm{~ns}),{ }^{34}$ and perylene $(8.9 \mathrm{~ns}) .{ }^{35}$ It is noteworthy that the lifetime of pyrene is significantly longer than that of naphthalene in spite of the pyrene's larger molecular size and level density. The mixing with the $S_{2}{ }^{1} B_{2 u}$ state might be shortening the lifetime of the $S_{1}{ }^{1} B_{3 u}$ state. However, the energy gap between the $S_{1}$ and $S_{2}$ states is almost the same for pyrene and naphthalene. The difference in their lifetimes, therefore, cannot be attributed to vibronic coupling between the $S_{1}$ and $S_{2}$ states. Another possibility is the potential energy curve. The obtained rotational constants of the $S_{1}$ state are similar to those of the $S_{0}$ state and the change in geometrical structure (displacement) is thought to be small. We suppose that the slope of the potential energy curve (distortion) is slightly shallower in the $S_{1}$ state of naphthalene. In pyrene, the slope is nearly the same in both the $S_{1}$ and $S_{0}$ states. In this case, the nonadiabatic Franck-Condon overlap is especially small and the $S_{1} \rightarrow S_{0}$ IC is expected to be minimal.

IVR is generally efficient in large PAHs because many vibronic levels are considerably mixed by the Coriolis inter- action or anharmonic interaction (Fermi resonance). The threshold excess energy depends on the molecular size or the number of normal modes because the interaction strength is approximately proportional to the coupling level density. The threshold energies are 3000,2122 , and $1300 \mathrm{~cm}^{-1}$ for benzene, ${ }^{36}$ naphthalene, ${ }^{37}$ and anthracene, ${ }^{33}$ respectively. In pyrene, IVR is remarkable in the vibronic level with an excess energy of larger than $730 \mathrm{~cm}^{-1}$. Radiationless transitions are often enhanced by IVR so that the fluorescence lifetime becomes shorter at high-vibrational levels. In pyrene, however, this enhancement is not significant and the decrease in lifetime with excess energy is relatively small.

\section{SUMMARY}

We have analyzed vibronic structure in the $S_{0}{ }^{1} A_{g}$ and $S_{1}{ }^{1} B_{3 u}$ states of the isolated pyrene molecule by fluorescence excitation and dispersed fluorescence spectra, as well as by ab initio calculation. The vibrational energies of the $S_{0}$ and $S_{1}$ states are similar and totally symmetric $a_{g}$ vibronic bands are weaker than the $0_{0}^{0}$ band. This suggests that the stable geometrical structures of pyrene are almost identical in both states. The $b_{1 g}$ bands were observed with appreciable intensity through vibronic interaction with the $S_{2}{ }^{1} B_{2 u}$ state. The rotational structure has been analyzed using ultrahighresolution fluorescence excitation spectroscopy. We accurately determined the rotational constants at the zerovibrational levels of the $S_{0}{ }^{1} A_{g}$ and $S_{1}{ }^{1} B_{3 u}$ states. It has been shown that the molecule is planar and that its geometrical structure does not significantly change upon electronic excitation. The vibronic coupling between the $S_{1}$ zero-vibrational level and $S_{0}$ high-vibrational levels is very small and the IC to the $S_{0}$ state becomes very slow. Predissociation does not occur in the $S_{1}$ state of PAHs, and ISC is also considered to be very slow. Consequently, all of the possible radiationless transitions are slow and fluorescence lifetime is accordingly very long in the $S_{1}$ state of the isolated pyrene molecule. The IVR rate is expected to be proportional to the density of coupling vibrational levels and the broad emission can be observed in the dispersed fluorescence spectrum even for the excitation of a low excess energy level at $730 \mathrm{~cm}^{-1}$.

\section{ACKNOWLEDGMENTS}

This work was supported by the JSPS Asian CORE program on Frontiers of Materials, Photo, and Theoretical Molecular Sciences, and also by the Joint Studies Program (2008-2009) of the Institute for Molecular Science. The authors are grateful to Professor Hiroki Nakamura (Institute for Molecular Science, Japan) and Professor Yuh-Lin Wang (Institute of Atomic and Molecular Science, R.O.C.). The authors thank to M. Aoyama, M. Kondo, T. Yano, N. Mizutani, and M. Suzui (Equipment Development Center, Institute for Molecular Science, Japan), and K. Mitsui and N. Okada (Advanced Technology Center, National Astronomical Observatory, Japan) for creating up a mirror setup for high-efficiency fluorescence collection.

\footnotetext{
${ }^{1}$ A. Bree and V. V. B. Vilkos, Spectrochim. Acta, Part A 27, 2333 (1971).

${ }^{2}$ W. H. van Leeuwen, J. Langelaar, and J. D. W. van Voorst, Chem. Phys. Lett. 13, 622 (1972).
} 
${ }^{3}$ L. Peter and G. Vaubel, Chem. Phys. Lett. 21, 158 (1973).

${ }^{4}$ F. W. Langkilde, E. W. Thulstrup, and J. Michl, J. Chem. Phys. 78, 3372 (1983).

${ }^{5}$ A. Bree, A. Leyderman, P. R. Salvi, and C. Taliani, Chem. Phys. 110, 211 (1986).

${ }^{6}$ C. M. Jones and S. A. Asher, J. Chem. Phys. 89, 2649 (1988).

${ }^{7}$ T. Kamisuki, H. Kataoka, and S. Maeda, Spectrochim. Acta, Part A 54, 277 (1998).

${ }^{8}$ J. Clairemidi, P. Bréchignac, G. Moreels, and D. Pautet, Planet. Space Sci. 52, 761 (2004).

${ }^{9}$ E. A. Mangle and M. R. Topp, J. Phys. Chem. 90, 802 (1986).

${ }^{10}$ N. Ohta, H. Baba, and G. Marconi, Chem. Phys. Lett. 133, 222 (1987).

${ }^{11}$ N. A. Borisevich, L. B. Vodovatov, G. G. D'yachenko, V. A. Petukhov, and M. A. Semyonov, J. Appl. Spectrosc. 62, 482 (1995).

${ }^{12}$ N. A. Borisevich, L. B. Vodovatov, G. G. D'yachenko, V. A. Petukhov, and M. A. Semyonov, Opt. Spectrosc. 78, 213 (1995).

${ }^{13}$ G. Rouillé, S. Krasnokutski, F. Huisken, T. Henning, O. Sukhorukov, and A. Staicu, J. Chem. Phys. 120, 6028 (2004).

${ }^{14}$ J. R. Platt, J. Chem. Phys. 17, 484 (1949).

${ }^{15}$ S. Gerstenkorn and P. Luc, Atlas du Spectre d'Absorption de la Molecúle D'Iode (CNRS, Paris, 1978).

${ }^{16}$ G. Orlandi and W. Siebrand, J. Chem. Phys. 58, 4513 (1973).

${ }^{17}$ K. Ohno, Chem. Phys. Lett. 64, 560 (1979).

${ }^{18}$ K. Ohno, J. Chem. Phys. 95, 5524 (1991).

${ }^{19}$ F. Negri and G. Orlandi, Theor. Comput. Chem. 16, 5129 (2005).

${ }^{20}$ M. J. Frisch, G. W. Trucks, H. B. Schlegel et al., GAUSSIAN 03, Revision
B.05, Gaussian, Inc., Pittsburgh PA, 2003.

${ }^{21}$ C. W. Bauschlicher and S. R. Langhoff, Spectrochim. Acta, Part A 53, 1225 (1997).

${ }^{22}$ J. K. G. Watson, in Vibrational Spectra and Structure, edited by J. R. Durig (Dekker, New York, 1977), Vol. 6, Chap. 1.

${ }^{23}$ M. A. El-Sayed, J. Chem. Phys. 38, 2834 (1963).

${ }^{24}$ W. Siebrand, J. Chem. Phys. 46, 440 (1967).

${ }^{25}$ S. H. Lin, J. Chem. Phys. 44, 3759 (1966).

${ }^{26}$ C. H. Chin, H. Y.-J. Shin, H.-W. Wang, Y.-L. Chen, C.-C. Wang, S. H. Lin, and M. Hayashi, J. Chin. Chem. Soc. (Taipei) 53, 131 (2006).

${ }^{27}$ M. Bixon and J. Jortner, J. Chem. Phys. 48, 715 (1968).

${ }^{28}$ H. Hirano and T. Azumi, Chem. Phys. Lett. 86, 109 (1982).

${ }^{29}$ N. I. Nijegorodov and W. S. Downey, J. Phys. Chem. 98, 5639 (1994).

${ }^{30}$ A. Bree, R. A. Kydd, T. N. Misra, and V. V. B. Vilkos, Spectrochim. Acta, Part A 27, 2315 (1971).

${ }^{31}$ P. R. Salvi, E. Castellucci, P. Foggi, and R. Quacquarini, Chem. Phys. 83, 345 (1984).

${ }^{32}$ S. Okajima, B. E. Forch, and E. C. Lim, J. Phys. Chem. 87, 4571 (1983).

${ }^{33}$ W. R. Lambert, P. M. Felker, and A. H. Zewail, J. Chem. Phys. 81, 2209 (1984).

${ }^{34}$ A. Amirav, U. Even, and J. Jortner, J. Chem. Phys. 75, 3770 (1981).

${ }^{35}$ A. J. Kaziska, S. A. Wittmeyer, A. L. Motyka, and M. R. Topp, Chem. Phys. Lett. 154, 199 (1989).

${ }^{36}$ K. G. Spears and S. A. Rice, J. Chem. Phys. 55, 5561 (1971).

${ }^{37}$ S. M. Beck, J. B. Hopkins, D. E. Powers, and R. E. Smalley, J. Chem. Phys. 74, 43 (1981). 